\title{
Processus urbains
}

Alain Bourdin

Recibido 2018.07.16 ::: Aceptado 2018.07.20

DOI: 10.5821/palimpsesto. 18.5685

a thématique de l'urbanisme provisoire n'est pas nouvelle, mais elle restait marginale et semble aujourd'hui en passe de s'imposer. De quoi s'agit il?

Si l'on crée un morceau de ville nouveau, celui-ci va mettre du temps à atteindre sa " vitesse de croisière " et pendant une période d'autant plus longue que l'opération est importante, prévaudra une situation provisoire, marquée par la faiblesse du nombre d'habitants, la rareté des services, dans certains pays la présence d'une voirie bordée de terres en friches, etc. On a mis longtemps à considérer que cela pouvait poser un problème. Ainsi a-t-on, en France développé une partie de la ville nouvelle de Cergy Pontoise avant que n'arrive le réseau express régional (métro express) alors que ce dernier était l'élément central du concept de cette ville nouvelle. A l'époque, aucune mesure spécifique n'a été prise pour rendre la ville habitable pendant cette période. Or, dans un nouvel établissement humain, les phases pionnières sont essentielles. La création d'une culture négative chez les premiers habitants, ou un affaiblissement de l'attractivité auront des conséquences néfastes à long terme. Au contraire, les premières phases peuvent faciliter la suite. J'ai travaillé sur un projet balnéaire (pour une dizaine de milliers d'habitants) dont la première version prévoyait la création du centre ville en fin de réalisation (dernière tranche). Si cela paraît une évidence économique car les commerces et les services ont besoin de clients, c'est une erreur majeure sur le plan de l'urbanisme et de ce qui y joue toujours un grand rôle : la satisfaction des usagers et l'image que porte un projet. Dans le cas présent, nous avions proposé de créer dés le début des éléments de centralité qui joueraient le rôle de " germes de ville ", quitte à ce qu'ils soient financièrement portés par le maître d'ouvrage, qui avait toutes les chances de regagner ultérieurement (meilleures ventes) ce qu'il perdrait à ce moment.

Le succès de cette thématique est encore plus fort lorsqu'il s'agit d'intervenir sur la ville existante, en particulier pendant les périodes ou de vastes espaces, friches industrielles, anciens grands équipements tels que des hôpitaux, etc., restent inoccupés. On a vu se développer toutes sortes de démarches visant à donner une utilisation et une vie provisoire à de tels espaces. Cette occupation provisoire, qui peut aller jusqu'à un coté "village Potemkine ", reste souvent centrée sur elle même plus que sur l'avenir : s'il a existé -en particulier en pays germaniquesdes expériences qui correspondaient à l'idée de " germe ", la mode actuelle consiste plutôt à favoriser des expériences supposée innovantes sur le plan social, des activités artistiques, ou encore de loisirs. L'écologie, l'agriculture urbaine, les expériences participatives, les ateliers de création y sont habituellement présents. Autrement dit cela appartient à la même famille que la mise en scène des chantiers (et l'éventuelle transformations de leurs clôtures en panneaux publicitaires), complètement indépendante du projet et de sa réalisation, et encore plus du fonctionnement et de la gestion du morceau de ville qui sera constitué. Cela ne change pas la logique du projet même si, ces dernières années, l'on a aussi admis qu'il existe un feed back et que l'on peut préciser la définition du projet ou l'ajuster (sans en changer les grandes lignes) en fonction de ce feed back.

Le jour ou l'urbanisme provisoire cessera de rester en marge du projet et ou des théories comme celles du " germe" (qui ne sont pas non plus nouvelles) seront poussées jusqu'à leur terme, une barrière sautera : celle du projet-clé de voute comme parti (ou dessein) initial auquel on doit finalement aboutir, même si c'est avec des déformations. Cela ne veut pas dire que le projet cessera d'exister, mais les processus seront considérés en eux même et non comme ce qui conduit au projet ou en est la conséquence, ou encore comme des phénomènes qui lui sont totalement étrangers, et forment une parenthsèse. La conduite des processus deviendra une question d'urbanisme autant que le projet lui-même. On voit bien ce que cela signifie quand on observe la place nouvelle que prend la question du commerce (et des rez-dechaussée) dans les projets urbains. Qui oserait encore dire qu'il suffit de créer des espaces commerciaux pour que le commerce vienne?
Je voudrais ici proposer quelques éléments de réflexion sur l'approche des processus en urbanisme. Pour ce faire je prendrai l'exemple des quartiers historiques. En effet ceux-ci reviennent au centre des préoccupations. Dans un pays comme la Chine, parce que les choix urbanistiques du pays sont en pleine mutation et que l'objectif de la ville durable associé aux évolutions idéologiques autour de la notion de patrimoine conduisent à changer le regard porté sur les quartiers anciens. Ailleurs, en partie à cause des interrogations sur la ville durable, mais aussi des phénomènes de " mise en tourisme » et de leurs excès, ainsi que, au moins dans le cas des villes moyennes, en raison de l'effondrement d'une partie du commerce de centre ville.

Mais je montrerai aussi que l'intérêt porté aux processus ne prend tout son sens que si l'on s'efforce de saisir l'état du système formé par le morceau de ville sur lequel on intervient (ce qui en général implique ses relations avec un système plus vaste).

\section{La gentrification: un exemple de} processus

Un quartier historique - ce qui selon les pays correspond à des frontières temporelles variables, mais va en tout cas jusqu'au $20^{\text {ème }}$ siècle - s'inscrit dans un ensemble de processus d'échelles différentes, des grandes tendances internationales, par exemple l'augmentation continue du tourisme urbain patrimonial, à des phénomènes très localisés, par exemple l'installation de quatre ou cinq boutiques vendant des vêtements au kilo dans un même morceaux de rue'; certains procèdent de l'action publique (opérations d'amélioration de l'habitat, créations de réseaux aujourd'hui plutôt la fibre optique que l'assainissement), d'autres de l'action de grands acteurs privés ou semi publics, d'autres enfin du croisement de l'action parfois quotidienne d'une multitude de petits acteurs. Certains d'entre eux sont très évidents, notamment ceux qui découlent de grandes opérations de restauration ou renouvellement urbain. D'autres, plus spontanés sont beaucoup plus difficiles à identifier et à observer. Une grande partie d'entre eux ont été réunis sous le terme de gentrification. Arrêtons nous un instant sur ce sujet pour réfléchir à ce qu'est un processus.

Le terme, gentrification, dans son usage commun, rassemble à peu près toutes les situations dans lesquelles une population plus aisée succède à une population modeste. Cela peut être par le simple jeu du marché (les propriétaires pauvres vendent à de plus riches) parce que les propriétaires augmentent le prix des loyers et font partir la population la plus pauvre, parce que des promoteurs transforment des parties entières du quartier, ou parce que des opérations publiques incitent à ce changement de population, par exemple à travers des aides financières à la restauration qui aboutissent à ce que dans les immeubles restaurés une nouvelle population plus aisée arrive soit comme locataire soit comme propriétaire.

Personnellement je considère que cette définition est beaucoup trop large et qu'il faut préciser les choses.

D'abord elle implique que l'on se trouve dans des situations qui permettent une forte augmentation de la valeur du sol et de l'immobilier. Ceci est généralement vrai dans les quartiers centraux des grandes villes ou des métropoles. Mais beaucoup moins dans des quartiers plus périphériques des mêmes villes, ce qui explique que le processus de gentrification y soit plus lent. Et cela peut devenir faux dans les quartiers historiques de petites villes qui connaissent des difficultés économiques.

Ensuite il faut faire la différence entre de purs phénomènes de marché et d'autres dont la dimension culturelle est importante. Tous les quartiers anciens -et récents- de Londres et Paris connaissent une 


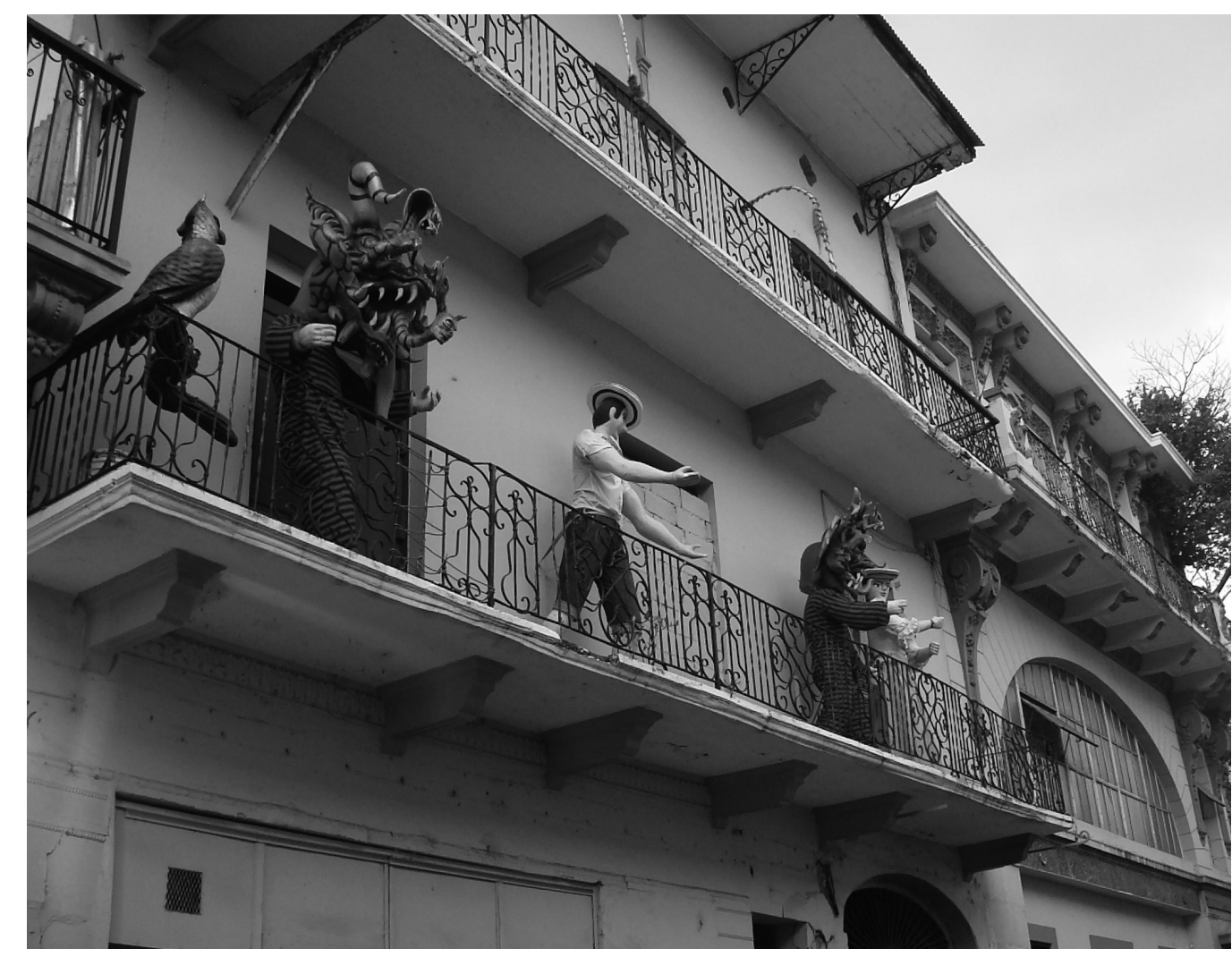

FIG. 2. Création de valeur culturelle à Panama la fin de leurs études. Ils marquent le quartier, mais la population habituelle qui reste beaucoup plus longtemps qu'eux continue à garder un contrôle sur son territoire.

Les jeunes professionnels peuvent s'investir très fortement dans le développement du quartier. Une partie d'entre eux appartient à des professions qui ont des raisons particulières de s'intéresser au patrimoine, par exemple des architectes. Certains d'entre eux vont rester mais beaucoup chercheront des quartiers moins urbains lorsqu'ils s'établiront vraiment et auront des enfants. Les familles établies appartiennent habituellement à la classe moyenne diplômée. Elles s'installent dans des quartiers en cours de gentrification mais pour y rester. Elles font monter la valeur générale du quartier sans pour autant être forcément préoccupées de réaliser des bénéfices immobiliers.

Une partie de ceux qui appartiennent à cette catégorie ou à la catégorie précédente n'ont pas de véritable objectif économique. S'ils sont locataires car aucun bénéfice ne s'offre à eux et même s'ils sont propriétaires et occupent un logement qui leur plait et qu'ils ne veulent pas quitter. A la limite il peut exister une gentrification qui se caractérise par une relativement faible création de valeur économique.

Un exemple intéressant à cet égard est celui du quartier du plateau à Montréal slide. Ce quartier, proche du campus urbain d'une grande université, est essentiellement peuplé par des immigrés, tous ayant de très hauts niveaux de diplômes mais des revenus plus limités, parce qu'ils sont en début de carrière ou qu'ils n'ont pas encore trouvé à Montréal un emploi correspondant vraiment à leur niveau de qualification. Ils sont très impliqués dans la vie du quartier et contribuent fortement à son animation. Ils interviennent dans les différents projets d'aménagement, et encore plus sur le fonctionnement des services publics: c'est le quartier de Montréal ou les parent sont les plus exigeants en ce qui concerne l'école publique (dans les quartiers pauvres beaucoup de gens ne s'intéressent pas à l'école et dans les quartiers riches on n'hésite pas à recourir à l'école privée). (ui même. Cela prend des formes un peu différentes selon qu'il s'agit d'un goût pour l'histoire, pour l'architecture ancienne ou pour ce qui nous vient du passé, et plus encore si c'est l'expression d'une vision du monde radicalement tournée vers le passé qui serait " bien meilleur que le présent ". de la part de sa population qui est qualifiée au niveau universitaire et dispose de revenus plus importants, au fonctionnement international du marché de l'immobilier. Ce phénomène n'a pas grand chose de culturel, sauf indirectement parce que Paris et Londres sont des villes où certains grands investisseurs (des pays du Golfe ou de la Russie par exemple) se plaisent.

Lorsque la sociologue Ruth Glass a inventé le terme de gentrification en 1964 à propos de Londres, elle voulait parler de tout autre chose. A l'époque, de jeunes diplômés des universités n'étaient pas tentés par la vie dans les quartiers pavillonnaires de la banlieue de Londres en compagnie de leurs semblables et loin de toutes les aménités du centre ville. Ils voulaient vivre près des cinémas, des théâtres, des restaurants, aller à des vernissages d'exposition dans les galeries etc. Bref mener une vie qui ressemble à celle des artistes connus, des professions libérales, de la grande bourgeoisie intellectuelle. De ce fait, ils se trouvaient un peu dans la position de la gentry (la petite noblesse) traditionnelle qui aurait bien voulu avoir le même genre de vie que la haute aristocratie mais n'en avait pas les moyens. D'où le terme gentrification. Par ailleurs, ces innovateurs qui voulaient vivre autrement que leurs parents étaient fortement portés à valoriser d'autres classes sociales que la bourgeoisie petite ou moyenne dont ils étaient issus, notamment la classe ouvrière. Ils considéraient donc qu'habiter un quartier à dominante ouvrière dans la proximité immédiate du centre de Londres était une bonne solution et ils ont évidemment contribué à transformer morphologiquement (en faisant des travaux) et socialement ces quartiers.

On doit accorder toute son importance à la dimension culturelle de la gentrification car c'est ce qui fait sa spécificité.

\section{Une création de valeur}

La gentrification est une création de valeur dans laquelle la valeur économique et la valeur culturelle se mêlent, cette dernière étant à l'origine du processus.

Lorsqu'un quartier ancien est investi par de nouveaux habitants uniquement parce que le logement y est moins cher qu'ailleurs (et que ce faisant il font monter les prix), il s'agit d'un pur phénomène de marché ; il n'y a pas d'intérêt à parler de gentrification, sauf si des critères plus culturels interviennent aussi dans ce choix.
Un autre intérêt porte sur le mode de vie du quartier. Une ambiance de village, l'existence de traditions particulières à la vie de quartier ou encore le gout de vivre avec des gens différents de soi. Cela peut déboucher sur la constitution de vastes espaces de convivialité sous l'impulsion des acteurs de la gentrification.

Un troisième intérêt concerne la forme urbaine : on peut préférer habiter dans le dédale des rues d'une médina plutôt que dans l'espace orthogonal des quartiers construits par les colonisateurs. Cela se traduit également par la recherche de l'authenticité : une vie quotidienne et des lieux authentique, par opposition aux lieux artificiels de la société de consommation. Sharon Zukin a montré à propos de New York que l'authenticité qui se veut refus des produits de grande consommation devient elle même un produit de grande consommation.

Une autre manière de s'intéresser à la forme urbaine et l'architecture consiste à y chercher -et y trouver des espaces pour y exercer des activités spécifiques, souvent artistiques, mais aussi de start up dans les nouvelles technologies. Cet intérêt a joué un rôle puissant pour la transformation d'espaces industriels ou portuaires qu'il n'était pas toujours facile de transformer en bureaux ou en logements. Et la présence d'artistes connus peut fortement transformer l'image d'un quartier qui, de ruine industrielle sans intérêt devient le lieu ou des artistes à la mode ont leur atelier, donc forcément positif. à la mode ont leur atelier, donc forcément positif. même rôle.

Outre la différence des intérêts culturels, on observe que les acteurs de la gentrification sont divers et que leur position dans le cycle de vie les conduit à avoir des attentes et élaborer des stratégies différentes.

Les étudiants s'installent assez volontiers dans des quartiers anciens qu'ils contribuent à gentrifier. En général ces quartiers se trouvent à proximité d'une université, comme par exemple à proximité de l'université américaine de Beyrouth. Ils y logent, participent facilement à la vie du quartier, lui trouvent de l'agrément. Mais il est rare qu'ils y restent après

\section{La complexité des processus}

Les voies empruntées par le processus de gentrification sont encore plus diverses que ne semble le dire ce qui précède. En effet on a surtout parlé de gentrification par le logement. On peut aussi évoquer la gentrification par l'activité. A la frontière est de Paris, à Montreuil, dans un quartier très populaire avec une population pauvre et en grande partie immigrée un grand nombre d'agences d'architecture se sont installées. Elles pouvaient y disposer à bas prix de locaux assez bien adaptés à leur activité et au type d'ambiances qu'aiment les architectes. Leur présence a fait évoluer le commerce -par exemple en suscitant la création d'un bon restaurant- et l'ambiance du quartier. La fonction résidentielle suit, d'ailleurs difficilement, et n'est en rien le moteur du processus. Dans le quartier latin de Montréal c'est non seulement la présence de l'université, mais le développement d'un ensemble de restaurants cafés etc. qui est moteur.

Ainsi pouvons nous finalement distinguer quatre grands types de gentrification (qu'il faudrait croiser avec le niveau de pression économique):

-La gentrification résidentielle qui va du bricolage réalisés par les occupants pour améliorer leur logement aux grosses opérations de restauration publiques et privées.

-La gentrification par les usages : quand ce sont essentiellement les activités et les usages qui changent et que cela entraîne un changement dans l'image du quartier, dans sa valeur économique et éventuellement dans sa composition résidentielle.

-La gentrification communautaire. Elle est typique de quartiers très connus comme Soho à New York mais elle peut exister dans d'autres endroits. Un premier scénario habituel est celui de la constitution d'un quartier très lié à une minorité (ethnique dans les pays à forte immigration). On peut avoir envie d'habiter Little Italy, Chinatown ou le quartier gay parce que c'est le quartier d'une communauté dont on se sent membre ou que l'on idéalise. Un autre scénario tient à l'impression villageoise que donne un quartier (une réalité ou une pure image) : on pense à l'exemple de 
Kreuzberg à Berlin. Enfin ce quartier communautaire peut être celui des intellectuels ou des artistes : on a envie d'habiter avec eux, parce que l'on se sent à tort ou à raison membre de leur groupe, ou parce qu'on les admire ou même par ce qu'on les envie.

-On se rapproche alors de la gentrification par l'art. Dans ce cas il importe moins de vivre avec la communauté des artistes ou de la côtoyer que de se trouver inscrit dans un processus de production artistique. Ce qui d'ailleurs implique moins la résidence que la fréquentation. Ce dernier type de gentrification est particulièrement important pour des friches industrielles portuaires ou ferroviaires, dont il s'agit de changer complètement l'image, et rapidement.

Car la gentrification est aussi une question de vitesse. Elle peut être très rapide et parfois elle le doit, en particulier quand il n'y a pas d'habitants au départ. Lorsqu'il y a des habitants la gentrification rapide s'avère génératrice de problèmes et très souvent violente à l'égard de ceux qui doivent partir.

Au contraire la gentrification lente présente souvent un caractère positif. Un des enjeux de l'urbanisme du patrimoine peut être de favoriser les processus de gentrification lente.

L'exemple de la gentrification dans les quartiers historiques illustre la complexité des processus qui font les transformations urbaines. Les militants peuvent les simplifier à outrance pour pouvoir faire entendre leurs revendications. Les chercheurs ne devraient pas le faire, mais il leur arrive de se laisser égarer par leurs convictions. Les urbanistes «n'ont pas le droit " de le faire. Leurs enjeu est de maîtriser ou au moins d'influencer ou d'accompagner les processus -et cela devient à mon sens une partie majeure du projet. Pour cela il faut les connaître de manière très précise, y compris dans les liens qu'ils entretiennent entre eux. Cela passe par un travail de terrain poussé et non par la référence à quelques grandes théories surplombantes (par exemple celle de la ville néo-libéral), quel que soit leur intérêt intellectuel.

\section{Écosystème et processus}

Mais la connaissance des processus implique -au moins idéalement- celle de l'état de l'éco-système que constitue l'entité sur laquelle on intervient (par exemple le quartier, lorsque celui-ci correspond à une réalité), à tout moment et en particulier à chaque grande étape, pour s'efforcer de trouver les moyens de maintenir ou de recréer son équilibre.

Toute entité urbaine peut être considérée de deux manières différentes : d'une part en tant qu'ensemble formant un système stable ou instable au moment où on l'observe, d'autre part à partir des processus auxquels elle est soumise. Les deux approches sont liées car les processus produisent de nouveaux états du système, mais la manière dont s'exercent les processus dépend des états successifs du système. Par exemple un quartier caractérisé par la domination d'une population de passage et des activités économiquement fragiles sera beaucoup plus vite transformé par le processus de gentrification qu'un quartier dans lequel on trouve des familles très enracinées (présentes depuis plusieurs génération) et un artisanat de haut niveau qui attire une nombreuse clientèle.

L'écosystème que forme un quartier comprend trois grandes dimensions : les objets matériels, principalement les bâtiments et l'espace public ; les activités et les services : entreprises, commerces, services publics; enfin les habitants. Ceux-ci peuvent avoir des rapports très différents à leur quartier : certains lui accordent une grande importance d'autres pas, certains ne s'intéressent qu'aux opération immobilières qu'ils vont y faire pour gagner de l'argent, d'autres sont amoureux du patrimoine ou de la vie traditionnelle ou de la culture spécifique de ce quartier, d'autres encore y viennent parce que c'est le bon endroit pour créer une activité nouvelle ou parce que c'est bien localisé dans l'agglomération. Tout cela constitue des modes d'occupation différents, qui concernent non seulement les habitants mais les usagers et les visiteurs. On peut aller à Venise pour visiter les principaux monuments en groupe et repartir parce que les hôtels sont chers, ou au contraire avoir envie de s'installer pendant beaucoup plus longtemps sur la Giudecca pour partager la vie des habitants.

Le problème est de savoir si ces modes d'occupation sont compatibles entre eux et s'ils le sont avec les caracteristiques des activités et services et avec celles des bâtiments et des espaces publics.

a question peut se poser à propos de la présence d'activités industrielles, ou encore d'activités de loisirs qui produisent du bruit et de l'animation très tard dans la nuit, dans un quartier historique plutôt destiné à I'habitat et dans lequel vit une population qui recherche la tranquillité.

Un exemple particulièrement intéressant est celui du développement de Rbnb et de ses conséquences dans les quartiers historiques européens. Deux éléments transforment l'écosystème du quartier : d'une part les propriétaires qui louaient des logements en mauvais état - par exemple les logements sous les toits des immeubles non restaurés dans le Maraisà une population modeste découvrent que l'on peut rembourser très vite d'importants travaux de restauration quand on a ensuite une clientèle Rbnb qui rapporte beaucoup plus que les locataires à l'année. Ils contribuent donc à faire partir la population la plus pauvre. D'autre part, les touristes de Rbnb ne s'intéressent qu'à un type de commerce (alimentaire pour des préparations rapide) et l'équilibre du commerce se trouve mis en difficulté.

On le voit donc, des phénomènes plus ou moins importants peuvent changer l'équilibre de l'écosystème et entraîner sa dislocation (que l'on peut analyser en mobilisant la théorie mathématique des catastrophes) ou au moins un fonctionnement de plus en plus difficile. II est donc important d'avoir une connaissance précise des états du système et de savoir définir les interventions, légères ou plus importantes qui permettent de rétablir l'équilibre ou d'en établir un nouveau. Pour prendre un seul exemple, la création d'une résidence d'étudiants peut être utilisée comme un outil pour rétablir l'équilibre dans un quartier où cohabitent une population traditionnelle assez pauvre et une population récente et plus aisée, dans la mesure ou par exemple les étudiants peuvent aller chez les commerçant qui correspondent à la population traditionnelle tout en ayant un mode de vie qui se rapproche de celui de la nouvelle population.

L'exemple de la gentrification dans les quartiers historiques illustre un changement que manifestent d'une autre manière l'urbanisme tactique ou l'urbanisme de gestion : désormais le projet ne peut plus faire l'impasse sur les processus qui font la ville au quotidien et doit prendre en compte les états successifs de l'écosystème. Cela signifie qu'il ne peut plus se désintéresser de ce qui fait la ville au quotidien - celui de la vie dans son épaisseur et pas celui du fantasme. Et encore plus que personne ne peut faire l'économie de la complexité et se contenter de doctrines et de slogans outrageusement simplificateurs.

Ce qui est justement le cas dans la rue qu'habite l'auteur à Paris.

ABSTRACT

Le succès de l'urbanisme provisoire témoigne d'une évolution plus gánérale. Elle consiste à donner plus d'importance aux processus qui font la ville et qui influencent considérablement la mise en œuvre des projets.

Cet article examine l'exemple de la gentrifcation pour montrer combien il est important de prendre en compte la diversité et la complexité de ces processus et de ne pas être simpliste. II montre aussi qu'un processus n'est vraiment compréhensible qu'à travers les états successifs du système dans lequel il se développe.

MOTS CLÉ: urbanisme, processus, gentrification

TRADUCCIÓN

El éxito del urbanismo provisional atestigua una evolución más general, que consiste en dar más importancia a los procesos que hacen la ciudad y que influyen considerablemente en la puesta en obra de los proyectos.

Este artículo examina el ejemplo de la gentrificación para mostrar hasta qué punto es necesario tener en cuenta la diversidad y la complejidad de estos procesos sin ser simplista. Al mismo tiempo, demuestra que un proceso sólo

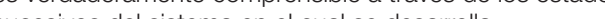

PALABRAS CLAVE: urbanismo, procesos, gentrificación.

ALAIN BOURDIN es Doctor en Sociología. Entre 2003 y 2011 fue director del Institut Français d'Urbanisme. 